OPEN

SUBJECT AREAS:

CORONARY ARTERY

DISEASE AND STABLE

ANGINA

NATURAL PRODUCTS

Received

9 September 2013

Accepted

15 January 2014

Published

4 February 2014

Correspondence and requests for materials should be addressed to

H.C. (chenhaojob@ yahoo.com) or M.L. (li.ming@ labinnovativemedicine. com)

\title{
Reconstitution of coronary vasculature by an active fraction of Geum japonicum in ischemic hearts
}

\author{
Hao Chen ${ }^{1,2}$, Lei Cheng ${ }^{2}$, Xiaoli Lin' ${ }^{3}$, Xiaping Zhou' ${ }^{1}$ Zhiming Cai' \& Ming Li ${ }^{3}$
}

\begin{abstract}
'The Second People's Hospital of Shenzhen, The First Affiliated Hospital of Shenzhen University, Shenzhen, PR China, ${ }^{2}$ Joint Laboratory For Regenerative Medicine, The Chinese University of Hong Kong, Shatin, Hong Kong, ${ }^{3}$ Laboratory of Innovative Medicine, Hong Kong.
\end{abstract}

Chronic coronary heart disease (cCHD) is characterized by atherosclerosis, which progressively narrows the coronary artery lumen and impairs myocardial blood flow. Restoration of occluded coronary vessels with newly formed collaterals remains an ideal therapeutic approach due to the need for redirecting blood flow into the ischemic heart. In this study, we investigated the effect of an active fraction isolated from Geum joponicum (AFGJ) on angiogenesis in cCHD hearts. Our results demonstrated that AFGJ not only enhanced capillary tube formation of endothelial cells, but also promoted the growth of new coronary collaterals (at the diameter $0.021-0.21 \mathrm{~mm}$ ) in the ischemic region of hearts in rat cCHD model. Our study also indicated that the growth of new collaterals in ischemic hearts resulted in improved functional recovery of the cCHD hearts as demonstrated by ECG and echocardiography analyses. These data suggest that AFGJ may provide a novel therapeutic method for effective treatment of cCHD.

$\mathrm{n}$ spite of the advances of modern medicine in the treatment of ischemic heart diseases, CHD including chronic CHD (cCHD) and myocardial infarction (MI) remains the leading cause of mortality in most developed countries $^{1,2}$. The development of $\mathrm{cCHD}$ due to coronary artery atherosclerosis is chronic and cumulative, consequently leading to the progressive narrowing or occlusion of affected coronary artery. When oxygen and glucose delivery become insufficient to meet the metabolic demands of the heart, a sequence of biochemical events will be triggered, leading to global myocardial injury. In addition, during vascular remodeling process due to chronic ischemia, the neovascularization to the ischemic heart tissues is insufficient to support the requirement of heart tissue compensation. Consequently, the cardiac myocytes become hypertrophied, and even $\mathrm{dead}^{3}$.

Recent advances in angioplasty and stenting, or coronary artery bypass grafting have targeted the $\mathrm{CHD}$ patients with major coronary artery atherosclerosis that limits blood flow in the large coronary arteries. However, patients having coronary plaques in small coronary arteries or complete blockage of the artery that cannot be crossed with the balloon are not amenable to balloon angioplasty and stenting or coronary bypass surgery. Although growth of new coronary collaterals into ischemic regions of the hearts in such patients would be beneficial, there are still no drugs or therapeutic modalities available that can promote these processes in such patients. Currently developed medications, such as statins, $\beta$-blockers, calcium channel blockers, ACE inhibitors, nitroglycerin, are predominantly focused on preventing the further narrowing of blood vessels which can only provide symptom relief ${ }^{4-6}$.

The inability of effective treatment of $\mathrm{cCHD}$ highlights the urgent need for a new method that can address the root pathology of the disease. Growth of new coronary collaterals including micro-arteries $(63-210 \mu \mathrm{m})$ and arterioles $(21-63 \mu \mathrm{m})$ into the ischemic territory of the hearts would restore the blood perfusion, which allows oxygen, nutrients, circulating stem cells and growth factors to be delivered to the ischemic region of the hearts supporting the repair of diseased hearts. In our previous studies, we demonstrated that an organic extract of Geum japonicum (EGJ) exerted dual effects on angiogenesis and cardiomyogenesis leading to substantial repair of infarcted hearts in animal MI models $s^{7,8}$. We further isolated a cardiogenic compound (cardiogenin) from EGJ that enhanced cardiogenic differentiation efficiency of mesenchymal stem cells in vitro and the substantial treatment of heart infarction in animal MI models mimicking the cardiomyogenesis activity of EGJ ${ }^{9}$. Another active fraction (Angio-T) was later isolated from EGJ with a demonstrated activity in promoting angiogenesis in cCHD rat model ${ }^{10}$. However, we had been puzzled by the instability of the angiogenic activity of Angio-T. Furthermore, the more detailed qualitative and quantitative studies of Angio-T promoted growth of new 
coronary collaterals in ischemic hearts are lacking in the previous studies. Therefore, in this study, we first aimed to work out a better method of isolating a stable angiogenic fraction from EGJ that can produce a well repeatable and stable effect on promoting the growth of new coronary collaterals in ischemic hearts. Secondly, we also performed more detailed qualitative and quantitative studies including the type, the quantitative analysis, and the distribution of the newly formed coronary collaterals with this stable angiogenic fraction.

\section{Results}

Isolation of AFGJ from G. japonicum Thunb. Var. Chinense F. Bolle. Reverse phase C18 column chromatography was used for the isolation of angiogenic AFGJ. Thin layer chromatography analysis demonstrated that AFGJ mainly comprised of polyphenols. The triterpenoids including the cardiogenic compound (cardiogenin) contained in the EGJ were removed ${ }^{9}$. The elution profile of AFGJ showed mainly 21 absorption peaks (Figure 1A), 5 of which showed approximately the same eluting time compared with those of Angio$\mathrm{T}$ (Figure 1B\&C). However, the heights and the areas of 3 (peaks 1, 2, 5 shown in Fig. 1C) of these 5 peaks are significantly higher and greater than the corresponding peaks of Angio-T. More importantly, some other peaks (peaks 3, 4 shown in Fig. 1C) were identified from AFGJ, but not from Angio-T. Similarly, a peak (peak 6 shown in Fig. 1C) was found in Angio-T, but not in AFGJ. These differences between the two fractions are probably due to a higher pressure and temperature used for isolation of Angio-T that made some compounds partially (peaks 1, 2 \& 5) or completely (peaks $3 \& 4$ ) degraded (Figure $1 \mathrm{~B} \& \mathrm{C}$ ). Activity study of AFGJ displayed a more potent and stable effect on promoting

A

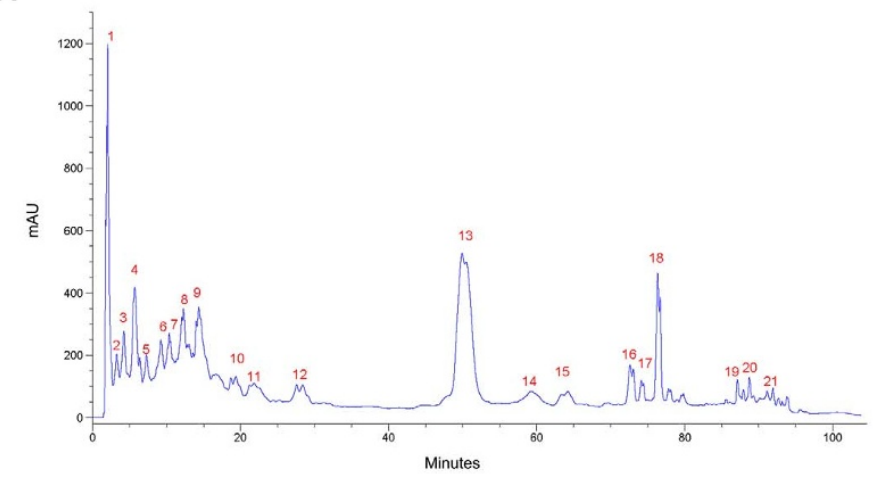

C

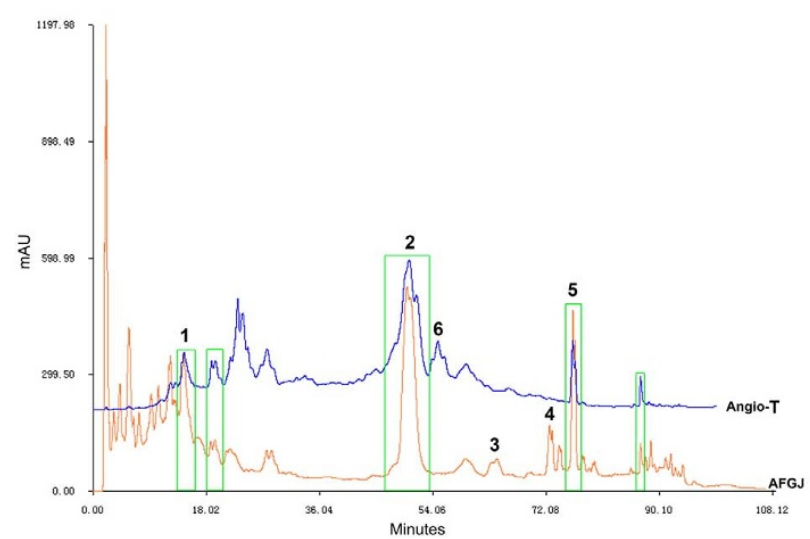

proliferation of HUVECs in vitro than did Angio-T. The results revealed that AFGJ could stimulate the proliferation of HUVEC in a dose-dependent manner. The proliferation rate in AFGJ-treated $(10-100 \mu \mathrm{g} / \mathrm{ml})$ increased by $\sim 1.5-2$ fold compared to that in nontreated control (Figure 1D).

AFGJ improves heart function in cCHD rat model. To investigate whether AFGJ enhanced neovascularization in ischemic heart would be translated into an improved functional performance of the diseased heart, ECG and echocardiography were used to measure the ischemia and heart function in both vehicle-treated control or AFGJ-treated hearts at different time points prior to/post treatments. It was found that similar normal waveforms of ECG were recorded before the surgery, however, the S-T segments of ECG diagram were significantly lowered after incomplete LAD ligation surgery indicating the ischemia of the hearts in experimental animals of both groups. Interestingly, the significantly lowered ST segments of ECG were progressively restored to the almost normal level 2 weeks post AFGJ treatment, indicating the restoration of blood perfusion to the ischemic hearts. By contrast, the lowered ST segments of ECG in the vehicle-treated hearts remained significantly depressed, suggesting the constant ischemia of the vehicle-treated hearts (Figure 2A). Quantitative analysis of the recorded ECG data demonstrated that the absolute value of ST segment depression was significantly restored in AFGJ-treated group (means \pm SD: $0.036 \pm$ $0.025 \mathrm{mV}$ ) compared to the values in vehicle-treated group (means \pm SD: $0.128 \pm 0.048 \mathrm{mV}$ ), in which $p<0.01$ (Figure 2B).

Echocardiography was used for evaluation of heart functional performance using TOSHIBA Xario ${ }^{\circledR}$ system. It was demonstrated that the left ventricle ejection fraction (LVEF) in the AFGJ treated

B

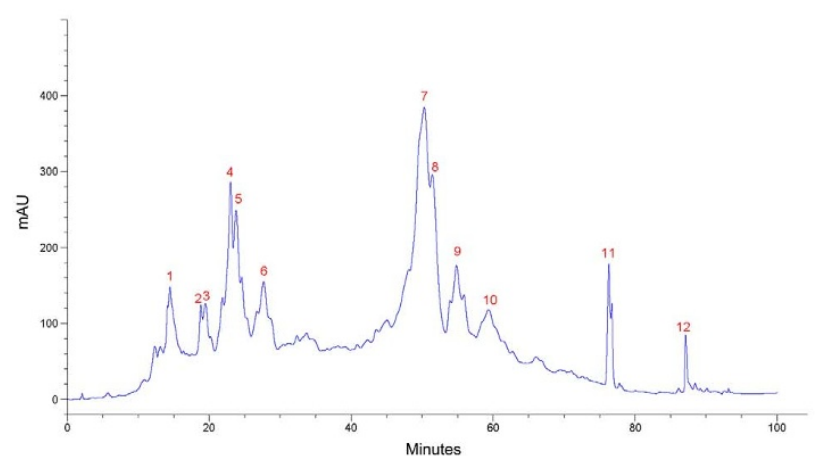

D

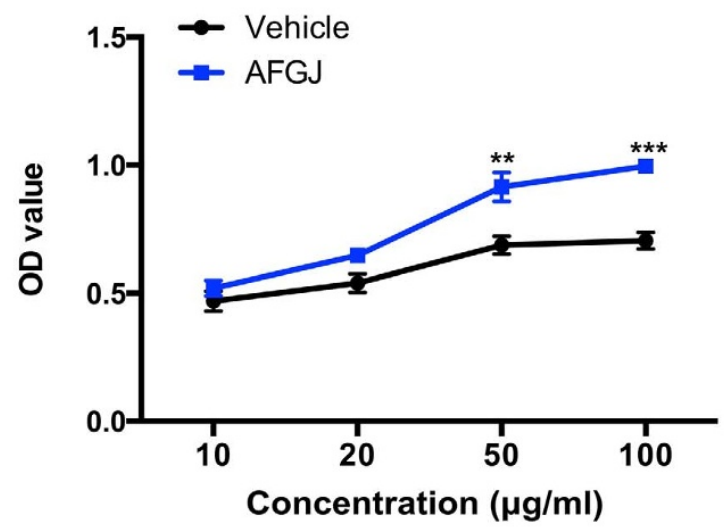

Figure 1 The HPLC finger-print chromatograms of AFGJ and its promoting effect on HUVECs growth. (A) A representative of AFGJ finger-print chromatogram. (B) Chromatogram of Angio-T. (C) Comparison of AFGJ and Angio-T. (D) AFGJ enhanced proliferation of HUVECs in a dosedependent manner by MTS assay. The experiments were repeated twice and total 9 measurements were analyzed. Values represent the mean \pm SEM. 
A

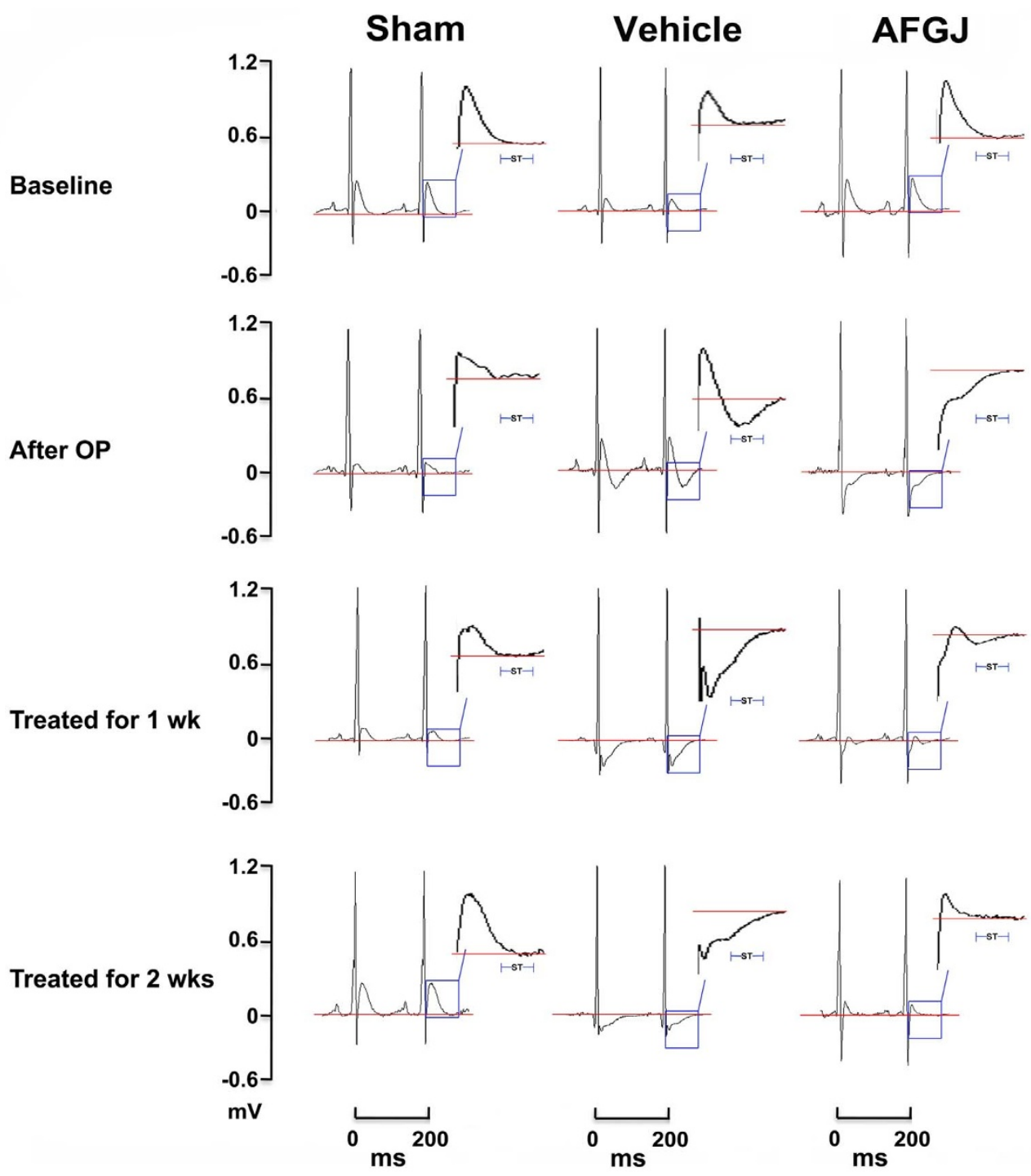

B
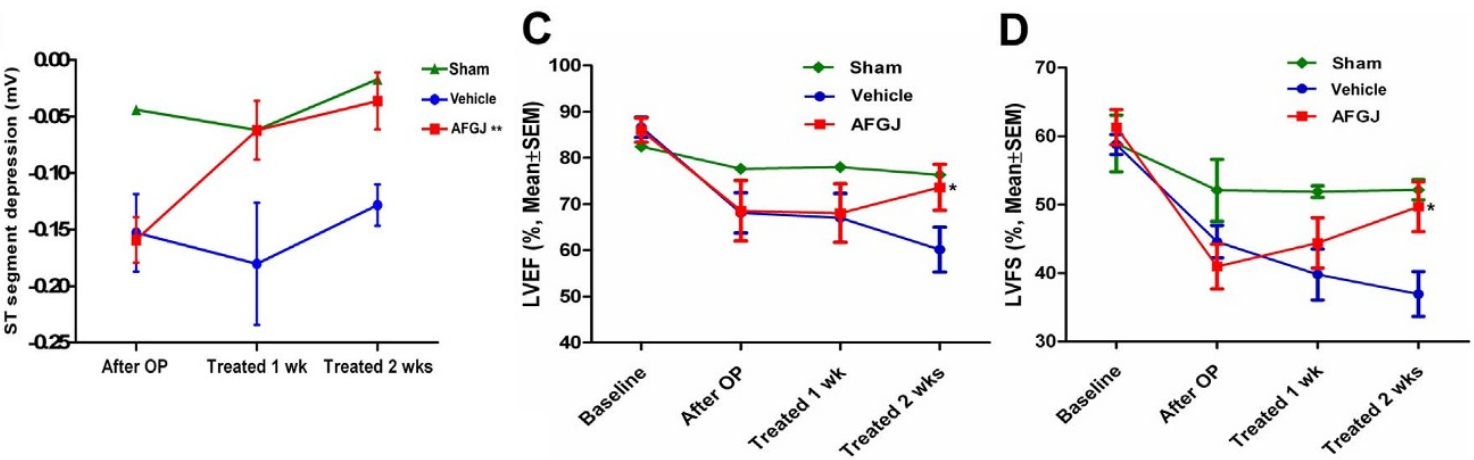

Figure 2 Electrocardiograms of CHD rats $(\mathbf{n}=7)$. (A) Two representative cycles of ECG recordings were taken from the sham-operated, vehicle and AFGJ treated experimental rats. Although the diagrams of ECG in sham-operated group, and in vehicle and AFGJ treated groups prior to LAD incomplete ligation showed typical normal ECG waves, the ST segments of the ECG diagrams were significantly depressed in vehicle and AFGJ treated animals after LAD incomplete ligation, indicating the successful induction of heart ischemia. Although the ST segment remained significantly depressed 1 or 2 weeks post ligation in the vehicle treated rats, the ST segment was progressively restored with time in the AFGJ treated rats. The Red line represents the baseline of ECG waveforms. (B) Mean data and statistics for ST segment depression. The baseline number was normalized as zero. Significant ischemia was present indicated by the lowered ST segment (decrease $>0.1 \mathrm{mV}$ ) in the vehicle group compared with that in the AFGJ treated group $(* * p<0.01)$. (C\&D) Echocardiography measurements $(n=7)$. LVFS was significantly improved in the AFGJ treated group compared with that in the vehicle group $(*, p<0.05)$. After LAD ligation, LVFS progressively lowered in the vehicle group while the LVFS in AFGJ treated was progressively restored from week 1 to 2. Similarly, the lowered LVEF was also progressively restored in AFGJ treated rats $(*, p<0.05)$. 

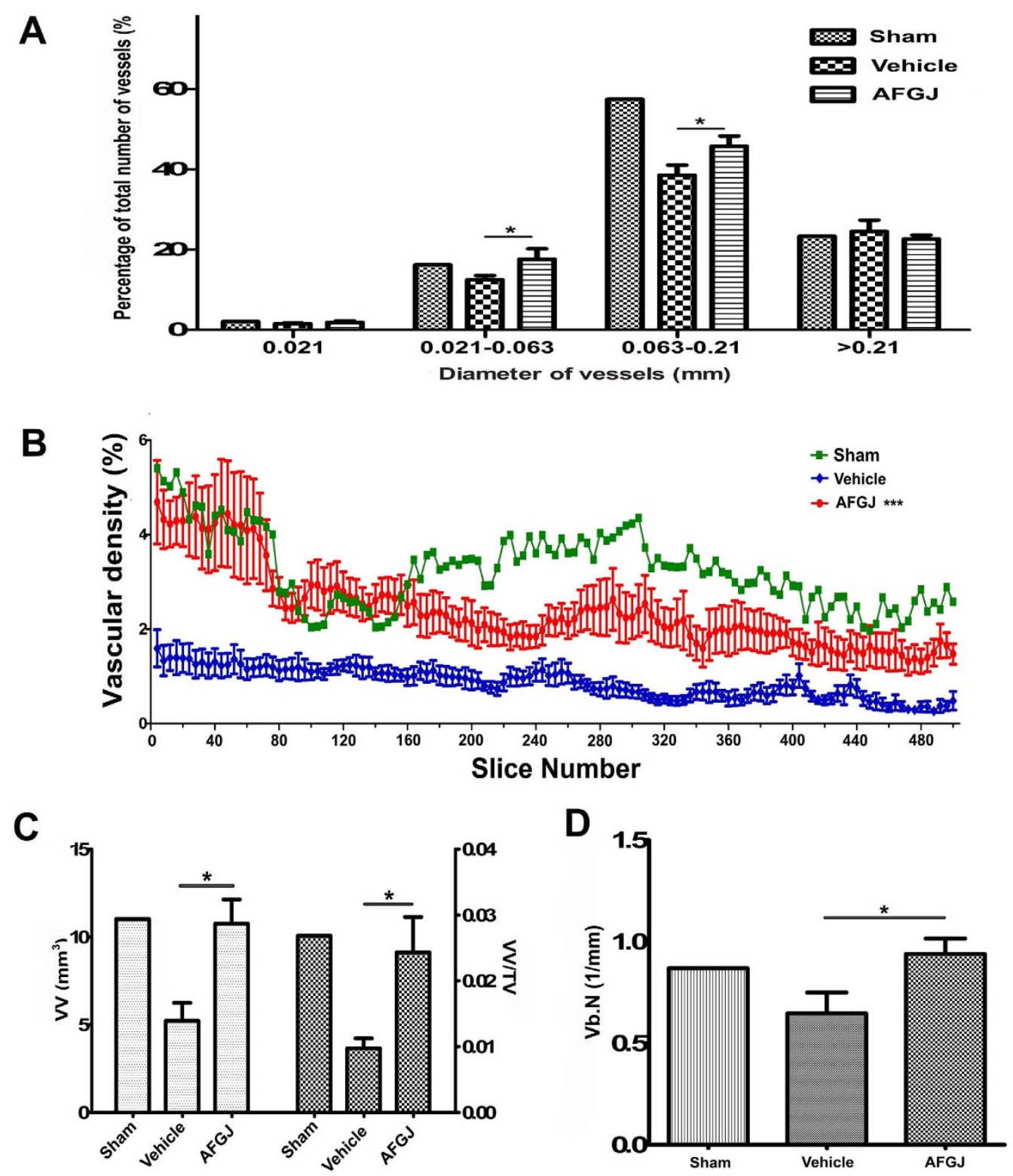

Figure $3 \mid$ MicroCT based quantitative analysis $(\mathbf{n}=6)$. (A) The distribution of different diameters of vessels. The densities of the arterioles $(21-63 \mu \mathrm{m})$ and the microvesesles $(63-210 \mu \mathrm{m})$ in the hearts of AFGJ-treated hearts were significantly higher than those in the hearts of vehicle-treated group $(*, p<$ 0.05). However, the densities of vessels (at diameters less than $21 \mu \mathrm{m}$ or greater than $210 \mu \mathrm{m}$ ) showed no significant difference between the two groups. (B) The representative $2 \mathrm{D}$ evaluation of unit area of vessels in ROI that was derived from 125 data sets of 500 slices. The result showed that the average density of vessels of AFGJ treated hearts was significantly higher than that of vehicle treated hearts (***, $p<0.001)$. (C) Quantitative volumetric measurements of heart vascular angiogenesis. The left $Y$-axis represents the vascular volume (VV) of ischemic area. VV was significantly increased in AFGJ treated hearts compared with that in vehicle treated hearts $\left({ }^{*}, p<0.05\right)$. The ratio of VV within total volume (TV) as illustrated by the right $\mathrm{Y}$-axis also increased in the AFGJ treated hearts compared with that in the vehicle treated group (*, $p<0.05)$. (D) Quantitative measurement of AFGJ induced therapeutic angiogenesis represented as vessel branching points. The bar graph showing the detailed information that the average number of segment cross-sectional vessels in AFGJ treated hearts was significantly increased compared with that in vehicle treated hearts $(*, p<0.05)$.

group showed significant improvement 2 weeks post treatment with mean \pm SEM $73.629 \pm 5$ versus $60.157 \pm 4.872$ (Figure $2 \mathrm{C}$ ). While left ventricle fraction shortening (LVFS) in the AFGJ-treated group was significantly higher than that in the vehicle-treated group 2 weeks post treatment with mean \pm SEM of $49.7 \pm 3.629$ versus $36.943 \pm 3.269$ (Figure 2D).

Coronary microvasculature: MicroCT based analysis. To demonstrate the direct evidence for AFGJ-enhanced small coronary collateral formation in ischemic hearts, MicroCT analysis was used to reconstruct the $3 \mathrm{D}$ vasculature of the ischemic hearts. We found that the AFGJ-treated hearts possessed more microvessels and collateral vessels compared to that in vehicle-treated hearts. This result provides the direct evidence for small coronary collateral formation in ischemic region of the $\mathrm{CCHD}$ hearts and the physical basis for substantial correction of heart ischemia and consequently the curative treatment of CCHD. More interestingly, the distribution analysis of average diameter of heart vessels (Figure 3A) demonstrated that the densities of arterioles $(21-63 \mu \mathrm{m})$ and microvesesles $(63-210 \mu \mathrm{m})$ in the hearts of AFGJ-treated group were significantly higher than those in vehicle-treated group, but close to those in sham-operated hearts. This result indicates that AFGJ treatment mainly stimulated the growth of small coronary arteries in ischemic hearts. This result is critically important for effective 
treatment of the patients, who have coronary plaques in small coronary arteries or a complete blockage of the artery that cannot be crossed with the balloon.

After analyzing whole heart images, the ischemic area (ROI/VOI) was determined. The quantitative analysis of small coronary collateral formation in the ischemic region of the hearts would provide the solid evidence for the beneficial effects of oral administration of AFGJ in CCHD. It was found that the amount of small coronary arteries including arterieos and microvessels in the AFGJ treated group was significantly increased compared to that in the vehicletreated group. Significantly higher vascular densities per unit area in ROI in the AFGJ-treated hearts were demonstrated, as compared with that in the vehicle-treated group (Figure 3B). Particularly, the density of vessels per unit area was remarkably higher in the AFGJ treated group than that in the vehicle-treated group in the first 100 slices within ischemic area (mean \pm SEM: $4.5 \pm 0.5$ versus $2 \pm 0.35$ ), which is approximately similar to the value in sham-operated hearts at the first 140 slices.

The quantification of microvessels of VOI was measured using a $3 \mathrm{D}$ evaluative method. The vascular volume (VV) of the AFGJ treated group was $10.754 \pm 1.386 \mathrm{~mm}^{3}$ compared with that of the vehicle-treated group of $5.217 \pm 1.032 \mathrm{~mm}^{3}$, which is consistent with the values of vascular densities in the same area. Furthermore, the $\mathrm{VV} / \mathrm{TV}$ in VOI showed a higher ratio of microvessel volume within total vessel volume in AFGJ treated hearts (mean \pm SEM: $0.024 \pm$ 0.005 ) than that in vehicle treated hearts (mean \pm SEM: $0.01 \pm$ 0.002), implying that AFGJ treatment significantly enhanced the growth of microvessels in the ischemic region of hearts (Figure 3C). More interestingly, both the values of VV and VV/TV in AFGJ treated ischemic hearts were virtually close to the values of non-ischemic hearts in sham operated group (mean: 10.754 versus 11.020 and 0.024 versus 0.027 ), indicating that AFGJ treatment effectively stimulated the growth of the coronary collaterals, especially the microvessels in ischemic region of the hearts. More importantly, the number of intersections between vessel and non-vessel components

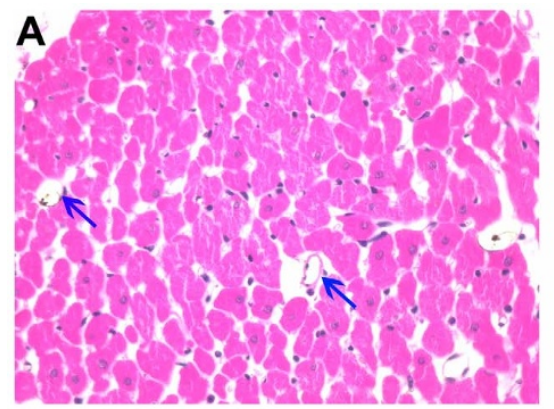

Sham

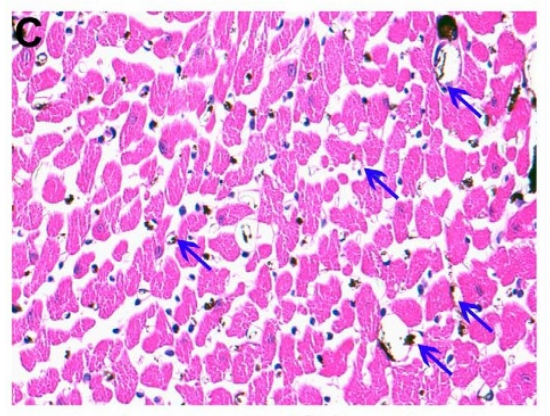

AFGJ per total length (vb. N) of vessels in VOI provided information on vessel branching points or segment cross-sectional vessel number (Figure 3D). There were substantially more branching points in the AFGJ treated group compared with that in the sham operated and vehicle treated group (mean \pm SEM: $0.9392 \pm 0.07615$ versus $0.8693 \pm 0.0001$ and $0.6462 \pm 0.1036$ ), suggesting that there were formation of new substantial collateral vessels in the AFGJ treated cCHD hearts.

In order to confirm the results obtained from MicroCT analysis, we further performed histological studies. Analysis of neovascularization in the ischemic hearts revealed that the density of small vessels and capillaries in AFGJ treated ischemic hearts was approximately $68.1 \pm 6.4$ per view field averaged from 5 randomly selected view fields of each slide and 6 slides in total from AFGJ treated hearts, which is virtually similar to the sham operated hearts $(74.5 \pm 6.7$ per view field). By contrast, significantly fewer blood vessels $(27.4 \pm 2.6$ per view field) were observed in the ischemic area of the vehicletreated control hearts (Figure 4). The data derived from histological quantitative analysis is consistent with that obtained from MicroCT quantitative analysis.

\section{Discussion}

Growth of new coronary collaterals in adults has been considered very limited. Therefore, cCHD is incurable and especially in cCHD patients with small coronary arteries narrowed/occluded. Currently, no treatment is proven to be effective other than symptomatic relief and general support ${ }^{4-6}$. Therefore, in the present study, we investigated the effect of AFGJ on angiogenesis through promoting the growth of small collateral arteries into the ischemic region of hearts in $\mathrm{CCHD}$ animal model.

We firstly revealed the similarities and differences in their compound composition between Angio-T and AFGJ. Activity test demonstrated that AFGJ could produce more stable and reproducible therapeutic angiogenesis compared with Angio-T. The underlying
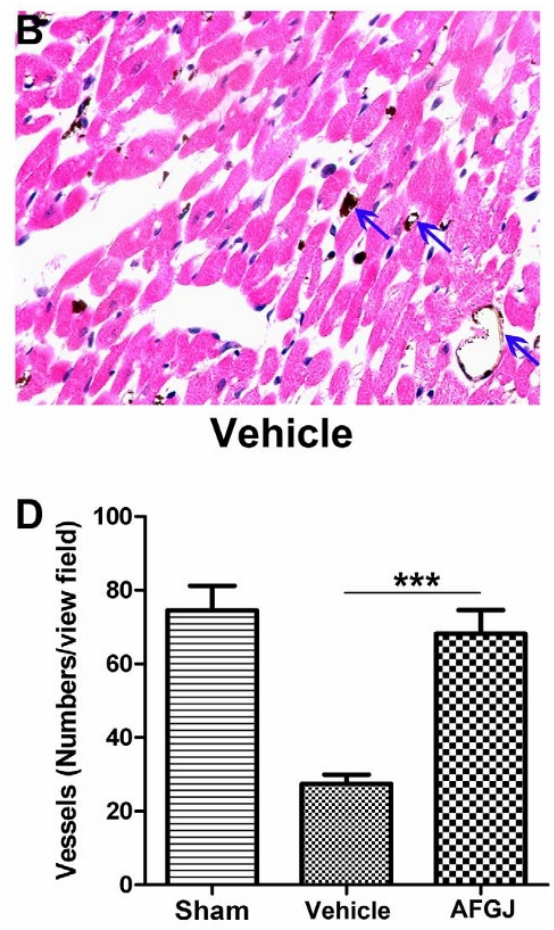

Figure 4 Analysis of vessel densities in ischemic region of cCHD hearts. Total blood vessels (blue arrows) in ischemic region of the vehicle treated hearts (B) were significantly less than those in sham-operated (A) and AFGJ treated hearts (C). The vascular density (D) in the AFGJ treated ischemic hearts is higher than that of vehicle treated hearts, but similar with that in sham-operated hearts. In each experiment, the microvessls were calculated from 5 random fields/mice. Data were presented as mean \pm SEM. $* * *, p<0.001$. 
player for sustaining the stable activity in promoting angiogenesis may reside in the stringent quality control in isolation of AFGJ that better reserved the active compounds, such as peaks $1-5$ (Fig. 1C) from partial or complete degradation as compared with Angio-T. Consequently, AFGJ rendered a more potent and stable effect on promoting capillary like structure formation of endothelial cells in vitro and coronary angiogenesis in vivo than did Angio-T. The AFGJ, which was isolated from EGJ extract with demonstrated dual actions of angiogenesis and cardiomyogenesis ${ }^{8,7}$ represents the angiogenic fraction of the dual active extract, which promotes the proliferation of vessel endothelial cells in low concentration and enhances vessel like-structure formation in vitro. However, AFGJ does not enhance the cardiogenic differentiation efficiency of bone marrow derived mesenchymal stem cells as cardiogenin, which is isolated from EGJ and represents the cardiogenic fraction of the EG) ${ }^{9}$.

To test this hypothesis of promoting growth of small coronary collateral arteries, we studied the therapeutic angiogenesis effect of the isolated AFGJ in a CCHD pre-clinical rat model. The results showed that oral administration of AFGJ to the experimental rats is effective in the CCHD animal model. Upon AFGJ treatment, the significantly lowered ST segments in ECG diagram due to heart ischemia were progressively restored and the heart functional performance was time-dependently improved. In consistence with these improvements, quantitative MicroCT results provided the solid evidence that the vascular density and collateral branching points in ischemic region of AFGJ treated hearts were significantly increased, which was further confirmed by quantitative histological analysis. MicroCT analysis provided a detailed visualization of vascular networks of the hearts that allows localizing the vessels and measuring vessel area/densities/diameter. The detailed analysis of MicroCT results showed that AFGJ treatment markedly increased the numbers of arterioles $(21-63 \mu \mathrm{m})$ and microvessels $(63-210 \mu \mathrm{m})$ that contributed to the formation of new collateral vascular network and the correction of heart ischemia. All these results indicate that AFGJ may provide alternative treatment of $\mathrm{CCHD}$ by addressing its root pathology, especially to those patients with small coronary arteries narrowed/occluded, which cannot be effectively treated by medication, invasive coronary angioplasty and stenting, or coronary artery bypass grafting. AFGJ-stimulated growth of new collateral vessels acts as a natural bypass of the narrowed/occluded coronary arteries that not only addresses the root pathology of cCHD but also effectively corrects the ischemia of the affected hearts.

It was known that the organic extract of Geum japonicum (EGJ) plays dual actions of promoting angiogenesis and inducing cardiomyogenesis ${ }^{7,8}$. It was found that a compound (cardiogenin) isolated from EGJ enhanced cardiogenic differentiation efficiency in cultured mesenchymal stem cells and induced myocardial regeneration in animal MI models mimicking the cardiomyogenic activity of the dual actions of EGJ ${ }^{9}$. The observed angiogenic promoting effect of AFGJ, which contains no cardiogenin and any noticeable cardiogenic activity (unpublished data), indicate that AFGJ is the angiogenic component of the dual actions of EGJ. As demonstrated, the elution profile of AFGJ showed mainly 21 absorption peaks comprising of polyphenols without containing triterpenoids. Compared with Angio-T, AFGJ contains 5 more and higher other common peaks. These differences may be probably due to a higher pressure and temperature used for isolation of Angio-T that makes some compounds degraded.

Taken together, AFGJ appears to promote therapeutic angiogenesis through induction of growth of new coronary collaterals (at the diameter $0.021-0.21 \mathrm{~mm}$ ) in ischemic hearts of the adult. The AFGJ induced growth of new collaterals in ischemic hearts is of therapeutic significance evidenced by the improved functional performance of the cCHD hearts. More importantly, the AFGJ induced growth of coronary collaterals into ischemic region of cCHD hearts should address the root pathology of the disease and provide a novel therapeutic method for effective/curative treatment of cCHD.

\section{Methods}

Animals. Male Sprague-Dawley (SD) rats of 250-300 g were obtained from the laboratory animal service center (LASEC) of the Chinese University of Hong Kong (CUHK). All animals handling protocols were approved by the Animal Research Ethics Committee of the Chinese University of Hong Kong under license from the government of the Hong Kong SAR. The investigation conforms to the Guide for the Care and Use of Laboratory Animals published by the US National Institutes of Health (NIH Publication No. 85-23, revised 1996).

Bioassay guided isolation of AFGJ. To avoid the potential toxicity of methanol and other residual solvents during the extraction of G. japonicum, isolation of active fractions was modified from previously described ${ }^{8}$ (Supplementary methods). The HPLC system used for isolation is an Agilent 1200 instrument (Agilent corp. Ltd., USA). For HPLC, $50 \mathrm{mg}$ AFGJ was dissolved respectively in $1 \mathrm{ml}$ methanol and $20 \mu \mathrm{l}$ sample was injected into reversed phase column (Sinochrom ODS-BP $5 \mathrm{um}, 4.6 \mathrm{~mm}$ $\times 150 \mathrm{~mm}$ [Dalian Elite Analytical instruments Co., Ltd.]). The detection wavelength was set to $254 \mathrm{~nm} .0 .1 \%$ Acetic acid $-\mathrm{H}_{2} \mathrm{O}$ (solvent A) and methanol (solvent B) were used as the mobile phase, starting with $0 \% \mathrm{~B}$ and increasing to $20 \% \mathrm{~B}(10 \mathrm{~min}), 30 \% \mathrm{~B}$ (10-40 min), 35\% B (40-50 min), 40\% B (50-60 min), 50\% B (60-70 min), 70\% B (70-80 $\mathrm{min})$ and $100 \% \mathrm{~B}(80-90 \mathrm{~min})$ with a solvent flow rate of $1 \mathrm{ml} / \mathrm{min}$ at $25^{\circ} \mathrm{C}$

Ambulatory ECG telemetry. ECG telemetry (PhysioTel CTA-F40) was implanted into the subcutaneous during operation to record electrocardiograms continuously ${ }^{11,12}$. Data acquisition and analysis was performed semiautomatically (Supplementary methods).

Chronic coronary heart disease rat model and treatment. The $\mathrm{CCHD}$ pre-clinical animal model was modified as previously described ${ }^{13}$. A left thoracotomy was performed and the pericardium was opened which lead to the exposure of LAD. An 80 silk suture was placed under the first branch of diagonal artery and a short segment of 11-0 silk suture was laid on the myocardium over the LAD. Then the 8-0 suture was tied up and the 11-0 silk suture was then drawn out gently to create a partially occluded LAD. The rats were monitored for 1 hour with continuous ECG recording 1 -week post surgery. For the test group $(n=7)$, prior to treatment, the rats were left to recover for 1 week then treated with AFGJ at $25 \mathrm{mg} / \mathrm{ml}$ and $2.5 \mathrm{ml}$ through P.O. For the control group $(n=7)$, the same procedures were conducted but treated with $\mathrm{H}_{2} \mathrm{O}$ instead of AFGJ. All specimens harvested were sectioned for histological and immunohistochemistry analyses.

Echocardiography. Each of 7 experimental rats received baseline echocardiograms before experimentation. Echocardiograms were recorded under controlled anesthesia (ketamine $(60 \mathrm{mg} / \mathrm{kg})$ and xylazine $[7.5 \mathrm{mg} / \mathrm{kg}])$ using a PST-AT6.5 MHz phasedarray transducer and a TOSHIBA Xario ${ }^{\circledR}$ system. For each measurement, at least three consecutive cardiac cycles were recorded. Left ventricular fractional shortening (FS) and ejection fraction (EF) were calculated by a program on the TOSHIBA Xario ${ }^{\circledR}$ system.

Coronary angio-perfusion and MicroCT analysis. To demonstrate the direct evidence of vascular bypass formation, MicroCT coronary angiography was performed (Supplementary methods). With this procedure, it provided clear visualization and quantitation of the newly formed coronary collateral vessels.

Effect of AFGJ on HUVECs. For cell proliferation analysis, MTS assay was used following the modified ATCC protocol ${ }^{14}$. HUVECs $\left(2 \times 10^{3} /\right.$ well $)$ were seeded into a 96-well culture plate with growth medium (Ham's F12K medium with 15\% FBS, 6 U/ $\mathrm{ml}$ heparin and $30 \mu \mathrm{g} / \mathrm{ml}$ endothelial growth factor supplements). After cell attachment, the medium was changed to Ham's F12K medium with $2 \%$ FBS for 12 hours. Cells were then treated with AFGJ of gradient concentrations 10, 20, 50, 100 and $200 \mu \mathrm{g} / \mathrm{ml}$, respectively for 36 hours. The OD value was measured by Tecan Sunrise plate reader (GmbH, Australia). Cell proliferation rates were statistically analyzed with Prism 5.0.

Statistics. All morphometric data were collected blindly. Results are presented as mean \pm SEM computed from mean measurements obtained from each rat heart. Statistical significance for comparison between two measurements was determined using the unpaired 2-tailed Student t-test. One way or two way ANOVA was used for evaluation of the 3 measurements. Values of $p<0.05$ were considered significant.

1. Isles, C. G. \& Paterson, J. R. Identifying patients at risk for coronary heart disease: implications from trials of lipid-lowering drug therapy. QJM 93, 567-574 (2000).

2. Delaney, E. K., Murchie, P., Lee, A. J., Ritchie, L. D. \& Campbell, N. C. Secondary prevention clinics for coronary heart disease: a 10-year follow-up of a randomised controlled trial in primary care. Heart 94, 1419-1423 (2008).

3. Shah, V. K. \& Shalia, K. K. Regeneration of Myocardium - Dawn of a New Era! $J$ Assoc Physicians India 57 (2009).

4. Aronow, W. S. Treatment of unstable angina pectoris/non-ST-segment elevation myocardial infarction in elderly patients. J Gerontol A Biol Sci Med Sci 58, M927-933 (2003)

5. Mason, R. P. \& Cockcroft, J. R. Targeting nitric oxide with drug therapy. J Clin Hypertens (Greenwich) 8, 40-52 (2006). 
6. Thakur, N. K. et al. Anti-atherosclerotic effect of beta-blocker with nitric oxidereleasing action on the severe atherosclerosis. J Cardiovasc Pharmacol 39, 298-309 (2002).

7. Li, M. et al. Repair of infarcted myocardium by an extract of Geum japonicum with dual effects on angiogenesis and myogenesis. Clinical chemistry $\mathbf{5 2}$ 1460-1468 (2006).

8. Cheung, W. L., Cheng, L., Liu, H., Gu, X. \& Li, M. The dual actions of angiogenesis and anti-apoptosis induced by an isolated fraction from Geum japonicum repair muscle ischemia. Archives of biochemistry and biophysics 459, 91-97 (2007).

9. Cheng, L. et al. A plant-derived remedy for repair of infarcted heart. PloS one 4, e4461 (2009).

10. Chen, H. et al. Reconstitution of coronary vasculature in ischemic hearts by plantderived angiogenic compounds. International journal of cardiology 156, 148-155 (2012).

11. Day, S. M. et al. Histidine button engineered into cardiac troponin I protects the ischemic and failing heart. Nat Med 12, 181-189 (2006).

12. Zimmermann, W. H. et al. Engineered heart tissue grafts improve systolic and diastolic function in infarcted rat hearts. Nat Med 12, 452-458 (2006).

13. Yoshinari, K., Yaoita, H., Maehara, K. \& Maruyama, Y. Different therapeutic responses to treadmill exercise of heart failure due to ischemia and infarction in rats. Cardiovasc Res 65, 457-468 (2005).

14. Vignais, M. L., Sadowski, H. B., Watling, D., Rogers, N. C. \& Gilman, M. Plateletderived growth factor induces phosphorylation of multiple JAK family kinases and STAT proteins. Mol Cell Biol 16, 1759-1769 (1996).

\section{Acknowledgments}

The work was supported by National Natural Science Foundation of China (No. 31100841), National Basic Research Program of China (2014CB745200), Shenzhen City Science and Technology Project Medicine and Health (No. 201202035), Shenzhen Projects of Science and Technology (GJHS20120702155016735), RGC grant (2140569) and CRF grant (3110044) in Hong Kong.

\section{Author contributions}

M.L. and H.C. wrote the main manuscript text. H.C. and L.C. performed the experiments and H.C. prepared the figures. X.L.L., X.P.Z. and Z.M.C. significantly contributed to manuscript revision. All authors reviewed the manuscript.

\section{Additional information}

Competing financial interests: The authors declare no competing financial interests.

How to cite this article: Chen, H. et al. Reconstitution of coronary vasculature by an active fraction of Geum japonicum in ischemic hearts. Sci. Rep. 4, 3962; DOI:10.1038/srep03962 (2014) (c) $(1)$ This work is licensed under a Creative Commons Attribution-
NonCommercial-NoDerivs 3.0 Unported license. To view a copy of this license,
visit http://creativecommons.org/licenses/by-nc-nd/3.0 